\title{
A toy model for generalised parton distributions
}

\author{
J.R. Cudell ${ }^{* \dagger}$, F. Bissey ${ }^{\ddagger}$ J. Cugnon ${ }^{\dagger}$, M. Jaminon ${ }^{\dagger}$, \\ J.P. Lansberg ${ }^{\dagger}$, and P. Stassart ${ }^{\dagger}$
}

November 6, 2018

pacs: $13.60 . \mathrm{Hb}, 13.60 . \mathrm{Fz}, 14.40 . \mathrm{Aq}, 12.38 . \mathrm{Aw}$.

keywords: parton distributions, GPD, bound states, gauge invariance.

\begin{abstract}
We give the results of a simple model for the diagonal and off-diagonal valence quark distributions of a pion. We show that structure can be implemented in a gauge-invariant manner. This explicit model questions the validity of the momentum sum rule, and gives an explicit counter-example to the WandzuraWilczek ansatz for twist-3 GPD's.
\end{abstract}

\section{Introduction}

We review the results on structure functions and generalised parton distributions (GPD's) that we obtained some time ago in [1]. Our purpose is to explore the simplest model of a hadron in order to have an explicit representation and some intuition for GPD's. Hence we have considered the simplest hadron, i.e. the pion, and concentrated on its valence-quark content. In order to further simplify the model, we took a $\pi^{0}$, although our twist-2 and twist-3 results remain true for $\pi^{ \pm}$, as the diagrams involving the direct $\gamma \pi^{ \pm}$coupling are suppressed by powers of $Q^{2}$.

*JR. Cudell@ulg.ac.be

†Physique théorique fondamentale, Dép. de Physique B5a, Univ. de Liège, B-4000 Liège, Belgium

†nstitute of Fundamental Sciences, Massey Univ., P.B. 11 222, Palmerston North, New Zealand 


\section{Diagonal structure}

\subsection{Structureless pions}

We must first consider the diagonal structure functions. In order to calculate them explicitly, we must make a model for the pion. The minimal requirement is that it is a pseudo-scalar made of a quark and an antiquark. Hence we must select the proper spin states, which is easily done through the use of a $\gamma_{5}$ vertex. The simplest assumption is then that the pion can be treated as a point-like particle, coupling to quarks via an effective 3-point vertex (shown in Fig प):

$$
\Gamma_{3}=i g \gamma_{5}
$$

with $g$ a coupling constant.

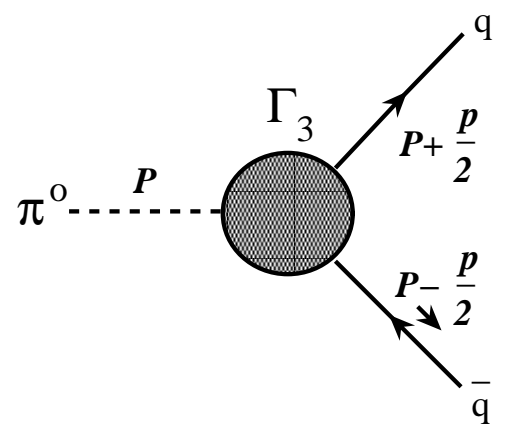

Figure 1: The lowest-order vertex $\pi^{0} q \bar{q}$.

The lowest-order approximation for the structure functions $F_{1}$ and $F_{2}$ then comes from the discontinuity $\operatorname{disc}\left(\mathcal{A}_{\mu \nu}\right)$ of the diagrams of Fig. 2] In order to make the calculation infrared finite (or at least to avoid the poles on the vertical lines of the diagrams of Fig. (2), we assume that the quarks are sufficiently massive:

$$
2 m_{q}>m_{\pi}
$$

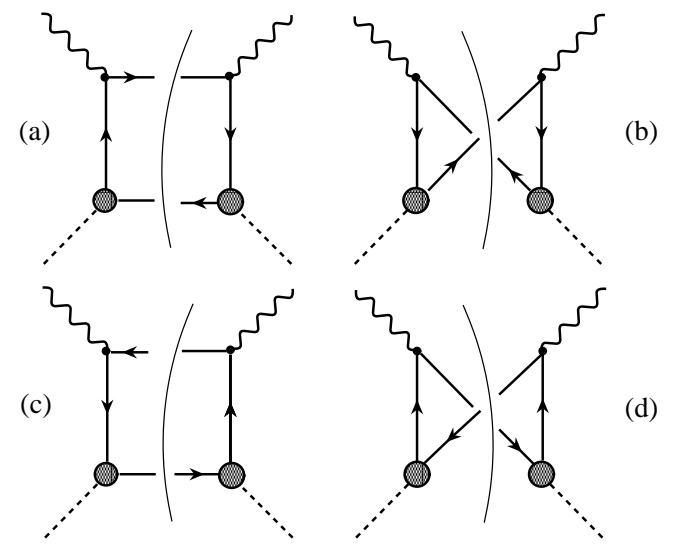

Figure 2: The four cut diagrams contributing to structure functions. 


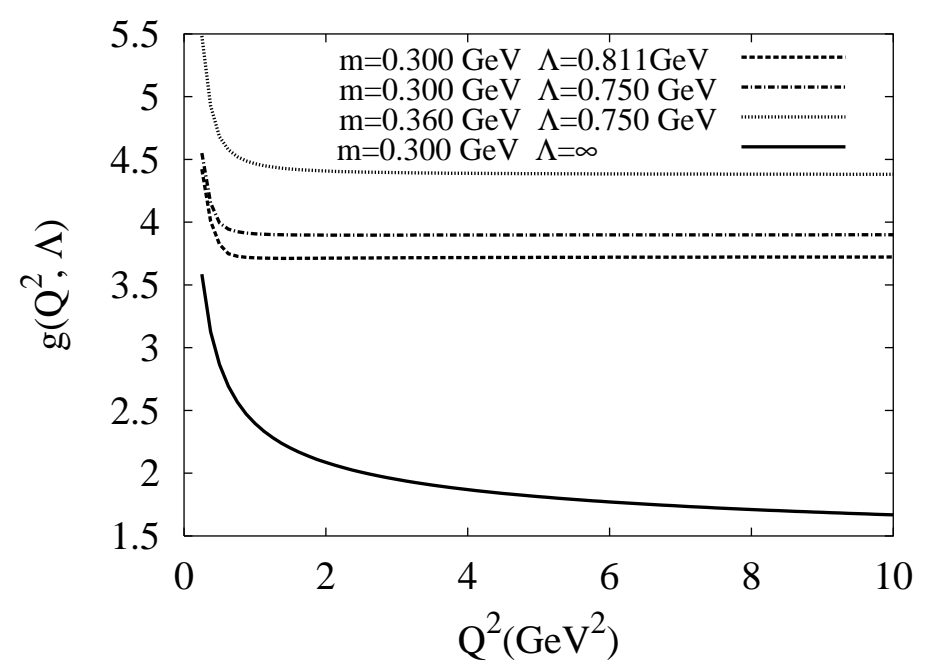

Figure 3: The coupling constant of the vertex.

(We shall take $m_{q} \approx 300 \mathrm{MeV}$ in the following.) The discontinuity is then obtained by putting the intermediate states on-shell. The answer one gets is explicitly gauge-invariant, and one may obtain the structure functions via the usual formula:

$$
\frac{1}{4 \pi} \operatorname{disc}\left(\mathcal{A}_{\mu \nu}\right)=\left(-g_{\mu \nu}+\frac{q_{\mu} q_{\nu}}{q^{2}}\right) F_{1}+\left(P_{\mu}-q_{\mu} \frac{P . q}{q^{2}}\right)\left(P_{\nu}-q_{\nu} \frac{P . q}{q^{2}}\right)(P . q) F_{2},
$$

with $P$ the momentum of the pion and $q$ that of the photon.

In the Bjorken limit, $Q^{2} \rightarrow \infty, x=Q^{2} /(2$ P.q) fixed, one obtains

$$
F_{1}=\frac{5 g^{2}}{24 \pi^{2}}\left[\log \left(\frac{(1-x) Q^{2}}{x M^{2}}\right)-\frac{m_{\pi}^{2}}{M^{2}} x(1-x)\right],
$$

with $M^{2}=m_{q}^{2}-m_{\pi}^{2} x(1-x)$.

One still needs however to determine the coupling $g$ of the vertex (11). In principle, this can be done via the electromagnetic form factor at $t=0$. We find it easier to use the Adler sum rule, which should be equivalent for the leading twist:

$$
\int_{0}^{1} d x \quad F_{1}\left(x, Q^{2}\right)=\frac{5}{18} .
$$

It amounts to saying that our pion is made of $u \bar{u}$ and $d \bar{d}$ with equal probability. It leads to a coupling $g$ given by the lower curve of Fig. 3. One obtains the Callan-Gross relation $F_{2}\left(x, Q^{2}\right)=2 x F_{1}\left(x, Q^{2}\right)$, and a prediction for $F_{1}$.

However, we see that, because $F_{1}$ has a logarithmic growth at fixed $g$, the normalisation condition actually makes $g$ run down as $1 / \log Q^{2}$. This is not consistent with the definition (II) of the vertex: $g$ can depend only on the variables entering the vertex, i.e. $P$ and $p$, and cannot depend directly on $Q^{2}$. 
Furthermore, although the diagrams of Fig. 2. a and 2. c have a probabilistic interpretation, they are not enhanced by a power of $Q^{2}$ with respect the interference graphs of Fig. 2. b and 2] d: we cannot define parton distributions. The reason for this is obvious: our pion does not have a structure.

\subsection{Structure and gauge invariance}

Guided again by simplicity, we shall assume that it is a good approximation to represent confinement effects by a cut on the square of the relative 4-momentum of the partons. The

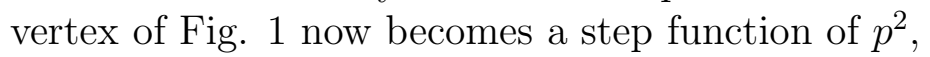

$$
\Gamma_{3}(P, p)=i g\left(Q^{2}, \Lambda^{2}\right) \gamma_{5} \theta\left(\left|p^{2}\right|<\Lambda^{2}\right)
$$

and we shall take $\Lambda \approx 0.8 \mathrm{GeV}$ (or $r_{\pi} \approx 0.25 \mathrm{fm}$ ). But the introduction of structure has a
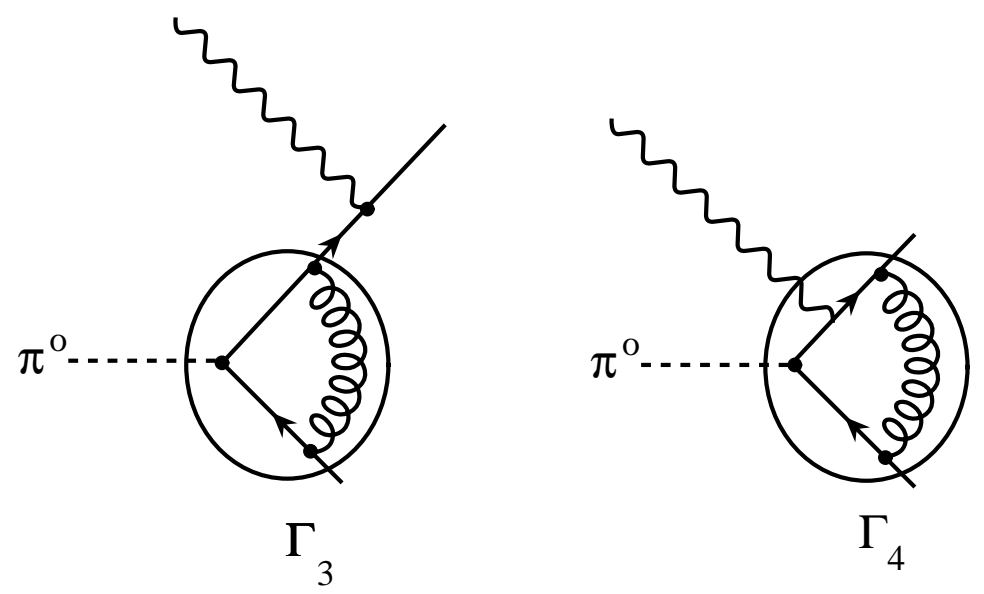

Figure 4: Illustration of the necessity of a 4-point vertex.

dire consequence: this simple modification is not sufficient as it breaks gauge invariance. This can easily be understood if we represent the vertex function by an exchange, as in Fig. 4. To get a complete set of diagrams leading to a gauge-invariant answer, on must include both diagrams in Fig. (4. The first diagram is analogous to a simple modification of the 3-point vertex, such as (6), whereas the second one can only be included in a 4-point vertex, as in Fig. 5 .

The latter is unknown, and we shall use a simple trick to model it. To analyse the problem, it is enough to consider one half of the cut amplitude, as in Fig. [6. We can define the usual Mandelstam variables as $\hat{t}=\left(P-p_{q}\right)^{2}$ and $\hat{u}=\left(P-p_{\bar{q}}\right)^{2}$. The cut on the relative momentum then amounts to a cut on $t$ for diagram [ [ a $\left(p^{2}=-m_{\pi}^{2}+2 m_{q}^{2}+2 t\right)$, whereas it is a cut on $u$ for diagram $6 \mathrm{~b}\left(p^{2}=-m_{\pi}^{2}+2 m_{q}^{2}+2 u\right)$. Hence both diagrams have different physical cuts, which gives rise to the gauge-invariance problem. The solution is then simple: one must invent a 4-point vertex such that both graphs are cut in the same 


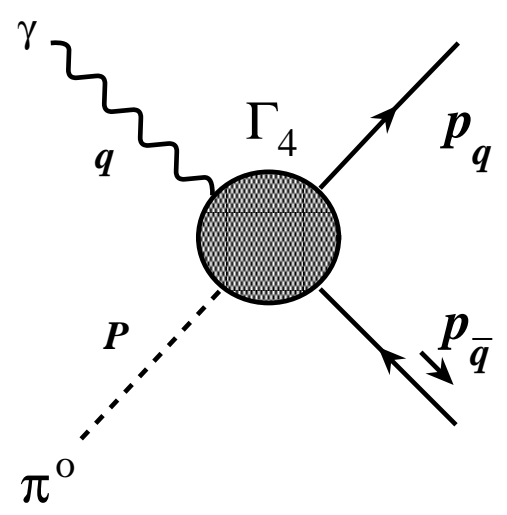

Figure 5: Representation of the 4-point vertex.

way. Hence, we need to multiply the sum of the two graphs of Fig. 6] by the same function and, to obtain (6), we must choose

$$
F(t, u)=\left[\theta\left(\left|-m_{\pi}^{2}+2 m_{q}^{2}+2 \hat{t}\right|<\Lambda^{2}\right)+\theta\left(\left|-m_{\pi}^{2}+2 m_{q}^{2}+2 \hat{u}\right|<\Lambda^{2}\right)\right] .
$$

The first term is the 3-point vertex (6), whereas the second term can be interpreted as a contribution from a new 4-point vertex, shown in Fig. 5 .

$$
\Gamma_{4}\left(p_{q}, p_{\bar{q}}, P, q\right)=g\left(Q^{2}, \Lambda^{2}\right) \theta\left(\left|-m_{\pi}^{2}+2 m_{q}^{2}+2 \hat{u}\right|<\Lambda^{2}\right) \frac{\gamma_{\mu}\left(\gamma \cdot\left(P-p_{\bar{q}}\right)+m_{q}\right) \gamma_{5}}{\left(P-p_{\bar{q}}\right)^{2}-m_{q}^{2}}
$$
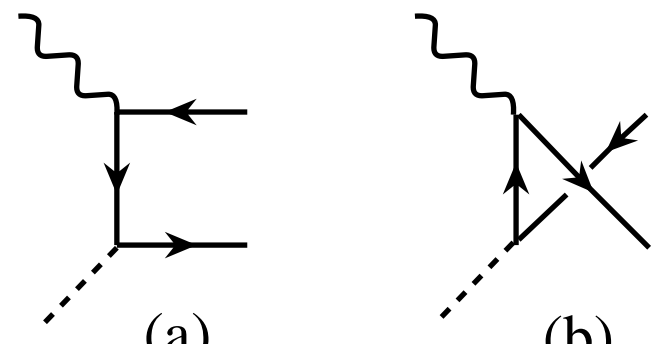

(a)

(b)

Figure 6: Two amplitudes of the cut.

One can then re-calculate $F_{1}$ and $F_{2}$, and normalise them again. The coupling constants are shown in Fig. 3. We see that $g$ can now be taken as a constant for values of $Q^{2}$ large enough for the Adler sum rule to hold. The curves for $F_{2}$ are given in Fig. 7 , for various choices of the cut-off $\Lambda$ and of the quark masses ${ }^{1}$.

One can see that $F_{2}$ is stable w.r.t. $Q^{2}$, so that the structure function seems a possible candidate for the initial valence quark distribution in a pion $u_{v}(x)=\bar{u}_{v}(x)=d_{v}(x)=$ $\bar{d}_{v}(x)=v(x)$, via the relation

$$
F_{1}=\frac{5}{9} v(x)
$$

\footnotetext{
${ }^{1}$ Please note that the curves are for $18 / 5 F_{2}$. The factor $18 / 5$ was missed in the first paper of [1].
} 
Note however that the condition $s>4 m_{q}^{2}$ leads to a cut on the values of $x$ which are allowed: whereas for no cut-off, $x$ can go to 1 in the limit $Q^{2} \rightarrow \infty$, it is limited to $x<1-\left(m_{q} / \Lambda\right)^{2}$ for finite $\Lambda$. Although the elimination of the interval close to $x=1$ is due to the fact that our cut-off is sharp, the suppression at large $x$ is reminiscent of that obtained in the covariant parton model [2. In this model, a vertex falling as $1 /\left(p^{2}\right)^{n}$ at large $p$ leads to a parton distribution that behaves like $(1-x)^{(n-1)}$.
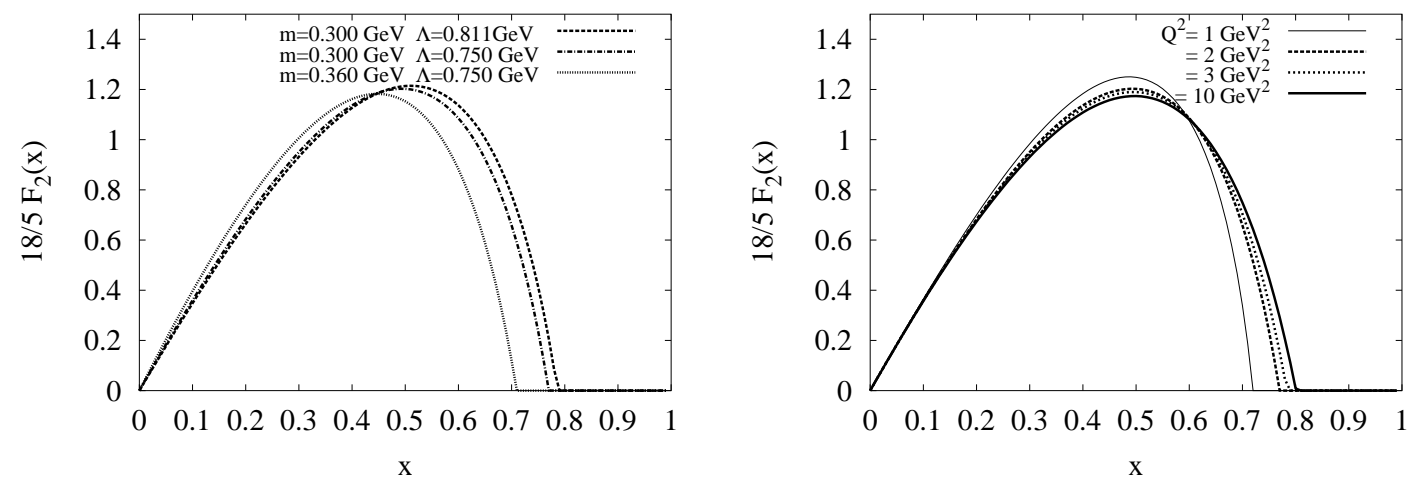

Figure 7: Results for the diagonal structure function. Left: for $Q^{2}=2 \mathrm{GeV}^{2}$, right: $m_{q}=0.3 \mathrm{GeV}, \Lambda=0.75 \mathrm{GeV}$.

This leads to one of the puzzles of this model. We can consider the average momentum carried by the quarks

$$
2\langle x\rangle=4 \int_{0}^{1} x v(x) d x=\frac{18}{5} \int_{0}^{1} F_{2}(x) d x .
$$

As we do not have gluons in the model, one would expect $2\langle x\rangle$ to be equal to 1 as $Q^{2} \rightarrow \infty$. However, we find that it is in fact significantly lower, as shown in Fig. 8. In fact, the momentum sum rule is correct either for $\Lambda=\infty$ (i.e. for structureless pions), or in the case $m_{q}<m_{\pi} / 2$ (as the infrared divergence can be re-absorbed in the normalisation).

Because the momentum sum rule is obeyed in the structureless case, and because high momenta are cut off in our model (or in the covariant parton model), it is obvious that $2\langle x\rangle$ must be smaller than 1 . There are two possible conclusions: the first is that the momentum sum rule holds only for free partons, so that it is not realised in our model, in which partons are always off-shell. But then physical quarks are always off-shell, so one may wonder if the sum rule is true. On the other hand, one may argue that the problem is that we did not consider cuts through the vertices, which would lead to a gluonic component. One must then understand why those cuts would have no effect on the sum rule if $m_{q}<m_{\pi} / 2$, whereas they would increase it by a factor 2 for higher masses. Whatever the scenario, the conclusion is that our calculation is reasonable for valence quarks. 


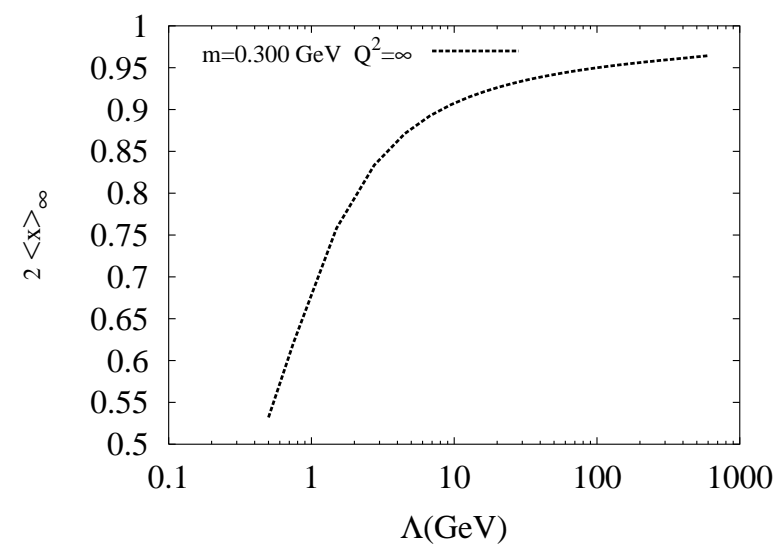

Figure 8: The average momentum carried by the quarks.

\subsection{Off-diagonal structure}

It is easy to extend our previous calculation to the off-diagonal case. Here, we shall again calculate the discontinuity of the amplitude, but with an off-diagonal kinematics: the external photons have incoming momentum $q_{1}$ and outgoing momentum $q_{2}$, whereas the pions have momenta $P_{1}$ and $P_{2}$. We define average momenta $P=\left(P_{1}+P_{2}\right) / 2$ and $q=\left(q_{1}+q_{2}\right) / 2$, and the momentum transfer $\Delta=P_{2}-P_{1}$. The Lorentz invariants of the process are $t=\Delta^{2}, Q^{2}=-q^{2}, x=Q^{2} /(2 P . q)$, and $\xi=\Delta . q /(2 P . q)$.

The calculation proceeds as in the diagonal case $^{2}$ : we calculate the discontinuity using the vertices $\Gamma_{3}$ and $\Gamma_{4}$ defined above. The answer is again gauge invariant, and can now be decomposed into 5 independent structures [3].

$$
\begin{aligned}
\frac{1}{4 \pi} \operatorname{disc}\left(\mathcal{A}_{\mu \nu}\right) & =-\mathcal{P}_{\mu \sigma} g^{\sigma \tau} \mathcal{P}_{\tau \nu} F_{1}+\frac{\mathcal{P}_{\mu \sigma} p^{\sigma} p^{\tau} \mathcal{P}_{\tau \nu}}{p \cdot q} F_{2} \\
& +\frac{\mathcal{P}_{\mu \sigma}\left(p^{\sigma}\left(\Delta^{\tau}-2 \xi p^{\tau}\right)+\left(\Delta^{\sigma}-2 \xi p^{\sigma}\right) p^{\tau}\right) \mathcal{P}_{\tau \nu}}{2 p \cdot q} F_{3} \\
& +\frac{\mathcal{P}_{\mu \sigma}\left(p^{\sigma}\left(\Delta^{\tau}-2 \xi p^{\tau}\right)-\left(\Delta^{\sigma}-2 \xi p^{\sigma}\right) p^{\tau}\right) \mathcal{P}_{\tau \nu}}{2 p \cdot q} F_{4} \\
& +\mathcal{P}_{\mu \sigma}\left(\Delta^{\sigma}-2 \xi p^{\sigma}\right)\left(\Delta^{\tau}-2 \xi p^{\tau}\right) \mathcal{P}_{\tau \nu} F_{5}
\end{aligned}
$$

where we have used the projector $\mathcal{P}_{\mu \nu}=g_{\mu \nu}-\frac{q_{2 \mu} q_{1 \nu}}{q_{1} \cdot q_{2}}$. For neutral pions, the structure functions $F_{i}$ that parameterize the discontinuity can be directly related to the GPD's $H$, $H^{3}$ and $\tilde{H}^{3}[3$ to twist-3 accuracy:

$$
\begin{aligned}
& \frac{1}{2 \pi} F_{1}=H, \\
& \frac{1}{2 \pi} F_{2}=2 x H+\mathcal{O}\left(1 / Q^{2}\right),
\end{aligned}
$$

\footnotetext{
${ }^{2}$ although we simplify the results by setting the pion mass to zero in the off-diagonal case.
} 


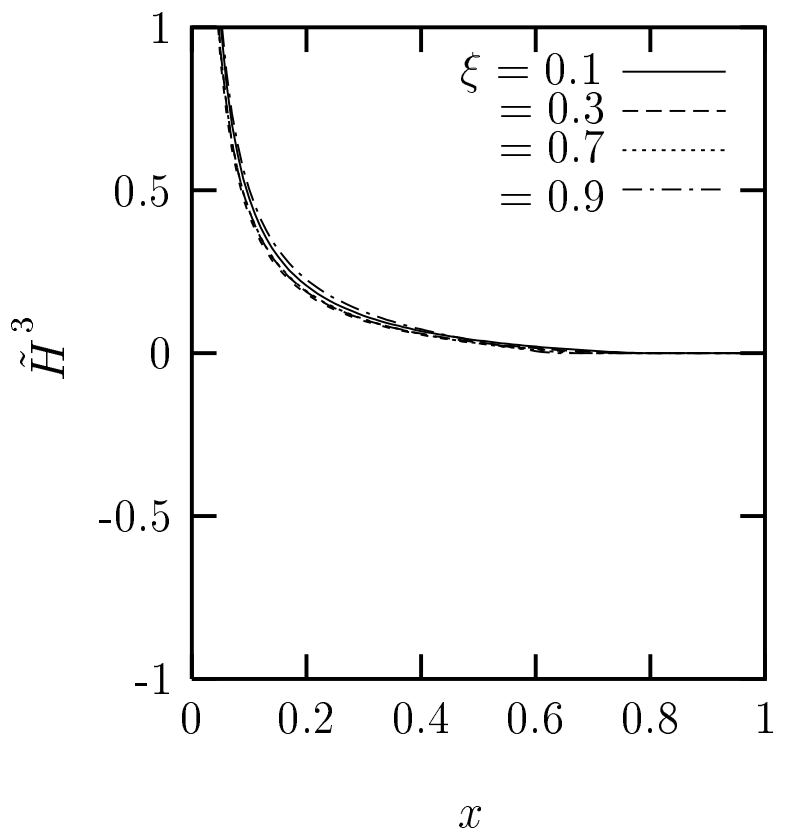

Figure 9: Our prediction for the valence component of $\tilde{H}^{3}$, for $Q^{2}=10 \mathrm{GeV}^{2}, t=-0.1$ $\mathrm{GeV}^{2}$ and $m_{\pi}=0$.

$$
\begin{aligned}
\frac{1}{2 \pi} F_{3} & =\frac{2 x}{x^{2}-\xi^{2}}\left(H^{3} x^{2}+\tilde{H}^{3} \xi x-H \xi\right)+\mathcal{O}\left(1 / Q^{2}\right), \\
\frac{1}{2 \pi} F_{4} & =\frac{2 x}{x^{2}-\xi^{2}}\left(H^{3} \xi x+\tilde{H}^{3} x^{2}-H x\right)+\mathcal{O}\left(1 / Q^{2}\right), \\
\frac{1}{2 \pi} F_{5} & =\mathcal{O}\left(1 / Q^{2}\right) .
\end{aligned}
$$

Our calculation obeys Eqs. (13) and (16), and leads to definite predictions for $H, H^{3}$ and $\tilde{H}^{3}$. Before giving the explicit results, let us mention that we find explicit relations linking the twist-3 GPD's to $H$ :

$$
\begin{aligned}
H^{3} & =\frac{(x-1) \xi}{x\left(\xi^{2}-1\right)} H+\mathcal{O}\left(1 / Q^{2}\right) \\
\tilde{H}^{3} & =\frac{H^{3}}{\xi}+\mathcal{O}\left(1 / Q^{2}\right)
\end{aligned}
$$

Note that the polynomiality of the Mellin moments of $H, H^{3}$ and $\tilde{H}^{3}$, together with Eqs. (17) and (18), implies that $H$ must be a polynomial $P_{H}(\xi)$ multiplying $\xi^{2}-1$. We show in Fig. 9 our results for $\tilde{H}^{3}$. The fact that it is almost independent of $\xi$ shows that $P_{H}$ is very close to a constant.

Let us point out that the relations (17, 18) are an explicit counter-example to the Wandzura-Wilczek ansatz 3 , 4. Not only are they numerically different, but our calculated $H^{3}$ and $\tilde{H}^{3}$ do not suffer from discontinuities at $x=\xi$, contrarily to what the WandzuraWilczek ansatz predicts. 
We can finally turn to our predictions for $H$. Here we consider two regimes: deeply virtual Compton scattering $(\xi=-x)$, and elastic scattering $(\xi=0)$. First of all, we show in Fig. 10 that our ansatz is stable w.r.t. $Q^{2}$. It can presumably be used as the initial parton distribution, as in the diagonal case.

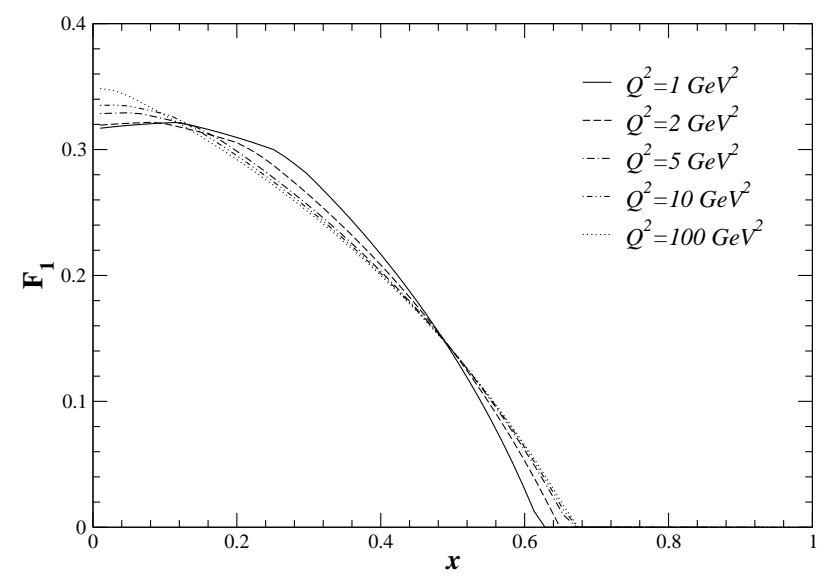

Figure 10: Our prediction for the valence component of $H$, in DVCS, as a function of $Q^{2}$, for $t=-0.1 \mathrm{GeV}^{2}, \Lambda=0.75 \mathrm{GeV}$ and $m_{\pi}=0$.

We can also examine the role of hadronic structure by comparing our prediction at $\Lambda=0.75 \mathrm{GeV}$ with that for a structureless pion $(\Lambda=\infty)$. We do this both in the DVCS and in the elastic cases in Fig. 111. We see that the structure of the pion makes an enormous difference. The cut-off in $x$ is in fact smaller than in the diagonal case, and the novelty is a rather large dependence on $t$. Hence we confirm that DVCS, and GPD's in general, will give us new information about hadronic structure.

\section{Conclusions}

We have built a very simple model for the pion, which goes beyond the spectator quark model. It implements all the symmetries of the problem, in particular gauge invariance. This toy model has enabled us to study explicitly initial valence quark (and antiquark) distributions, both in the diagonal and in the off-diagonal cases. We find that structure leads to corrections of order $m_{q} / \Lambda$ to the momentum sum rule in the diagonal case. Such effects might be attributed to the contributions of cuts in the vertices. Using this model in the off-diagonal case, we have shown that the Wandzura-Wilczek ansatz, which can be used to relate twist-3 GPD's to twist-2, is likely to be wrong. We in fact obtain new relations between the GPD's. We have also shown that binding effects lead to a rich structure for GPD's, which is not present in the case of point couplings. 

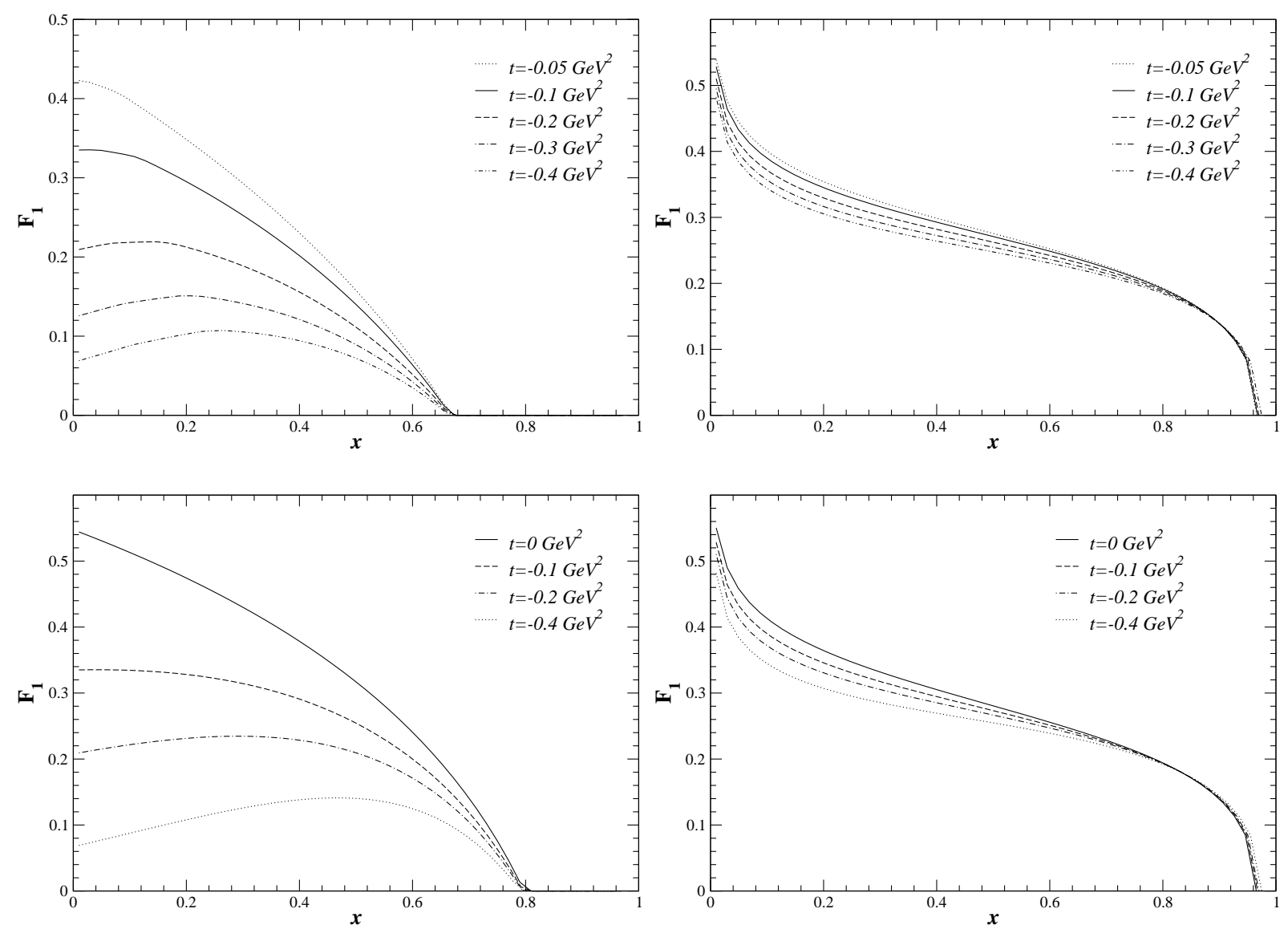

Figure 11: Our prediction for the valence component of $H$, in DVCS (top) and in elastic scattering (bottom), as a function of $t$, for $Q^{2}=10 \mathrm{GeV}^{2}$ and $m_{\pi}=0$. The left graphs are for $\Lambda=0.75 \mathrm{GeV}$ and the right ones for structureless pions.

\section{Acknowledgments}

We thank M.V. Polyakov and P.V. Landshoff for discussions. J.P.L. is a research fellow of the Institut Interuniversitaire des Sciences Nucléaires.

\section{References}

[1] F. Bissey, J. R. Cudell, J. Cugnon, J. P. Lansberg and P. Stassart, Phys. Lett. B 587 (2004) 189 arXiv:hep-ph/0310184; F. Bissey, J. R. Cudell, J. Cugnon, M. Jaminon, J. P. Lansberg and P. Stassart, Phys. Lett. B 547 (2002) 210 arXiv:hep-ph/0207107.

[2] P. V. Landshoff, J. C. Polkinghorne and R. D. Short, Nucl. Phys. B 28 (1971) 225;

P. V. Landshoff and D. M. Scott, Nucl. Phys. B 131 (1977) 172. 
[3] A. V. Belitsky, D. Müller, A. Kirchner and A. Schäfer, Phys. Rev. D 64 (2001) 116002 arXiv:hep-ph/0011314.

[4] S. Wandzura and F. Wilczek, Phys. Lett. B 72 (1977) 195. 